\title{
Calidad seminal de ovinos de pelo suplementados con Moringa oleifera (Moringaceae) y Trichanthera gigantea (Acanthaceae)
}

Marco A. Ramírez-Bautista ${ }^{a, b}$

Julio P. Ramón-Ugalde ${ }^{\text {a }}$

Edgar Aguilar-Urquizo ${ }^{\text {a }}$

William Cetzal-Ix ${ }^{b^{*}}$

Roberto Sanginés-García ${ }^{\text {a }}$

Álvaro E. Domínguez-Rebolledo ${ }^{\mathrm{c}}$

Ángel T. Piñeiro-Vázquez ${ }^{\mathrm{a}}$

a Tecnológico Nacional de México, Instituto Tecnológico de Conkal, División de Estudios de Posgrado e Investigación, Ave. Tecnológico s/n Conkal, 97345, Yucatán, México.

${ }^{\mathrm{b}}$ Tecnológico Nacional de México, Instituto Tecnológico de Chiná, Campeche, México.

c Instituto Nacional de Investigaciones Forestales, Agrícolas y Pecuarias, Campo Experimental Mocochá, Yucatán, México.

*Autor de correspondencia: rolito22@ hotmail.com

\section{Resumen:}

Se evalúo el efecto de la inclusión de Moringa oleifera Lam. y Trichanthera gigantea (Bonpl.) Nees en la dieta de ovinos de pelo (Pelibuey) sobre su calidad seminal. Durante 90 días, las dietas de 15 ovinos $(24 \mathrm{~kg} \pm 3.95)$ se dividieron en tres tratamientos: $\mathrm{T} 1$ : dieta integral con $40 \%$ de M. oleifera + pasto Taiwán (Pennisetum purpureum Schumach.), T2: dieta integral con $40 \%$ de T. gigantea + pasto Taiwán y T3: $40 \%$ alimento comercial + pasto Taiwán. A estos ovinos se les determinó la ganancia diaria de peso (GDP), rendimiento 
de la canal (RC), desarrollo testicular (DT) (determinado por el diámetro (AE) y la circunferencia escrotal (CE)); y a sus muestras de esperma se les evalúo el volumen, concentración, motilidad (CASA), viabilidad (SYBR-14/IP), actividad mitocondrial (J-C1) e integridad acrosomal (FITC-PSA). No se encontraron diferencias $(P>0.05)$ en la GDP y RC. Se encontraron diferencias $(P<0.05)$ en DT, T1 $(\mathrm{AE}=48.84 \pm 5.99 \mathrm{~mm}, \mathrm{CE}=26.48 \pm$ $1.13 \mathrm{~cm})$ y $\mathrm{T} 3(\mathrm{AE}=48.83 \pm 4.34 \mathrm{~mm}, \mathrm{C}=26.62 \pm 1.27 \mathrm{~cm})$ presentaron valores más elevados que $\mathrm{T} 2(\mathrm{AE}=44.57 \pm 5.59 \mathrm{~mm}, \mathrm{CE}=25.42 \pm 1.50 \mathrm{~cm})$; en la viabilidad, $\mathrm{T} 2(62.90$ $\pm 6.10 \%)$ y $\mathrm{T} 1(54.00 \pm 6.61 \%)$ poseen mayores porcentajes que T3 $(24.45 \pm 7.56 \%)$; en la motilidad, T1 $(93.9 \pm 2.1 \%)$ y T2 $(88.6 \pm 1.9 \%)$ tuvieron mayor porcentaje que T3 (71.9 $\pm 4.0 \%$ ). La inclusión de $M$. oleifera y $T$. gigantea en la dieta permite obtener una GDP, RC y DT similar al alimento comercial e incrementa más del $20 \%$ de las células espermáticas viables; también mejora algunos parámetros de motilidad, mejorando con esto el potencial reproductivo de los sementales.

Palabras clave: Células espermáticas viables, Motilidad, Pennisetum purpureum, Rendimiento de canal.

Recibido: 03/08/2018

Aceptado: 06/05/2019

\section{Introducción}

La elevada demanda de carne ovina obliga a los productores a mejorar todos sus procesos encaminados a reducir los costos de producción, a incrementar los parámetros productivos y reproductivos que permitan aumentar la productividad por unidad de superficie, mejorar el bienestar animal, y a anteponer un enfoque sustentable que asegure la permanencia del sistema a través del tiempo ${ }^{(1)}$.

En las zonas tropicales el ambiente es determinante en la producción pecuaria y en especial la producción ovina, debido a que enfrenta entre otros factores una marcada estacionalidad en la producción de forraje obligando a los productores a implementar estrategias de suplementación (principalmente con alimento comercial) para mantener niveles óptimos de producción, lo que ha generado una dependencia alimentaria y un considerable aumento en los costos de producción ${ }^{(2)}$. Bajo este contexto, la utilización de especies arbóreas con potencial forrajero cobran relevancia en la alimentación animal ${ }^{(3,4,5)}$, debido a que su implementación permite generar estrategias de sustitución parcial de granos en la dieta ${ }^{(6)}$. Sin embargo, la utilización de especies arbóreas con potencial forrajero está condicionada por el elevado contenido de nutrimentos, tolerancia a la poda, producción constante de 
biomasa y contenido de compuestos secundarios que pueden afectar a los animales que los consumen $^{(7,8)}$. Esto último, es una de las principales razones que limitan su uso para la alimentación animal ${ }^{(9)}$; ya que puede generar efectos estrogénicos que inhiben, entre otros, el crecimiento y el mantenimiento del sistema reproductivo. En el sistema reproductivo de los machos las afectaciones por presencia de compuestos secundarios pueden ser: mayor número de espermatozoides inmaduros con disminución significativa en la motilidad, disminución del lumen de los túbulos seminíferos, incremento del índice apoptótico de las células germinales, atrofia prostática y afección de la espermatogénesis ${ }^{(10,11)}$.

En este contexto, diversas especies como Moringa oleifera Lam. (Moringaceae) y Trichanthera gigantea (Bonpl.) Nees (Acanthaceae) son alternativas de suplemento alimenticio $^{(8)}$. A pesar de que se ha reportado en éstas la presencia de metabolitos secundarios; por ejemplo, García ${ }^{(12)}$ registró la presencia de fenoles, flavonoides y taninos que precipitan la proteína y taninos condensados; por su parte, Balercia et al ${ }^{(13)}$, Rajimakers et $a l^{(14)}$ y Ghasi et $a l^{(15)}$ observaron en $M$. oleifera y $T$. gigantea, la presencia de riboflavinas, ácido nicotínico, ácido fólico, piridoxina, proteínas, vitaminas A, B, C y E, así como aminoácidos y altos niveles de $\beta$-carotenos; el consumo de estos antioxidantes contribuye a mejorar la calidad seminal ya que eliminan radicales libres que se encuentran en el plasma seminal. Por tal razón, es necesario estudiar el efecto que estas plantas podrían generar en los animales que las consumen. Por lo anterior, el objetivo fue evaluar el efecto de la inclusión de M. oleifera y T. gigantea en la dieta de ovinos Pelibuey sobre la calidad seminal.

\section{Material y métodos}

El trabajo se desarrolló en el área de producción e investigación agrícola y pecuaria del Instituto Tecnológico de Conkal, Yucatán, México ( $21^{\circ} 05^{\prime} \mathrm{N}$ y $\left.89^{\circ} 32^{\prime} \mathrm{O}\right)$, que se encuentra a una altitud de $7 \mathrm{msnm}$, con clima tipo $\mathrm{AW}_{0}$, con precipitación de $900 \mathrm{~mm}$ anuales y temperatura promedio de $29^{\circ} \mathrm{C}$.

\section{Tratamientos, animales y manejo}

Se utilizaron 15 ovinos Pelibuey machos púberes $(6 \pm 0.5$ meses de edad), con un peso promedio de $24 \mathrm{~kg} \pm 3.95$ distribuidos completamente al azar en tres tratamientos $(\mathrm{n}=5)$ : $\mathrm{T} 1=$ dieta integral con $40 \%$ de M. oleifera + pasto Taiwán (Pennisetum purpureum Schumach.). $\mathrm{T} 2=$ dieta integral con $40 \%$ de Trichanthera gigantea + pasto Taiwán. T3= dieta integral + pasto Taiwán. La cantidad de alimento ofertada fue del $4.7 \%$ del peso vivo durante los 90 días que duro el experimento (los ajustes del consumo se realizaron cada siete días). El contenido de la composición porcentual de las dietas experimentales se puede observar en el Cuadro 1, y el análisis proximal en el Cuadro 2. 
Cuadro 1: Composición porcentual de las dietas experimentales

\begin{tabular}{llll}
\hline \multirow{2}{*}{ Componente } & \multicolumn{2}{l}{ Dietas $(\boldsymbol{\%})$} & T3 \\
\cline { 2 - 4 } & T1 & T2 & 0 \\
\hline Moringa & 16 & 0 & 0 \\
Trichanthera & 0 & 16 & 7 \\
Melaza & 4.24 & 3.72 & 3 \\
Minerales & 1.2 & 1.08 & 21 \\
Canola & 3.2 & 5.88 & 5 \\
Maíz & 8.28 & 8 & 4 \\
Sorgo & 7.08 & 5.32 & 60.0 \\
Pasto Taiwán & 60.0 & 60.0 & \\
\hline
\end{tabular}

T1: dieta integral con 40\% de M. oleifera + pasto Taiwán (Pennisetum purpureum Schumach.), T2: dieta integral con 40\% de T. gigantea + pasto Taiwán, y T3: alimento comercial + pasto Taiwán.

Cuadro 2: Análisis químico proximal de las dietas ofertadas

\begin{tabular}{llllll}
\hline $\begin{array}{l}\text { Dietas } \\
\text { y }\end{array}$ & $\begin{array}{l}\text { Materia } \\
\text { paca }\end{array}$ & $\begin{array}{l}\text { Proteína } \\
\text { cruda }\end{array}$ & $\begin{array}{l}\text { Fibra } \\
\text { detergente } \\
\text { neutra }\end{array}$ & $\begin{array}{l}\text { Fibra } \\
\text { detergente } \\
\text { ácida }\end{array}$ & Cenizas \\
\hline T1 & 90.83 & 13.81 & 20.22 & 16.18 & 1.01 \\
T2 & 91.54 & 14.01 & 26.27 & 14.21 & 0.95 \\
T3 & 98.82 & 23.16 & 22.83 & 13.47 & 0.98 \\
Pasto Taiwán & 90.96 & 8.00 & 67.26 & 42.21 & 0.97 \\
\hline
\end{tabular}

T1: dieta integral con 40\% de M. oleifera + pasto Taiwán (Pennisetum purpureum Schumach.), T2: dieta integral con 40\% de T. gigantea + pasto Taiwán, y T3: alimento comercial + pasto Taiwán

\section{Desarrollo de corderos}

Para determinar el desarrollo de los corderos se realizó el pesaje de los animales cada siete días, previo a cada pesaje los animales se sometieron a un período de $18 \mathrm{~h}$ de ayuno, se pesaron en una báscula T31P (OHAUS) de plataforma electrónica con capacidad de $300 \mathrm{~kg}$ con un margen de error de $0.1 \mathrm{~kg}$. Con el análisis de estos pesos se determinó la ganancia diaria de peso $^{(16)}$.

\section{Desarrollo testicular}

La medición de la circunferencia escrotal se realizó empleando una cinta métrica de plástico, mientras que para el registro del diámetro testicular se utilizó un vernier digital de la marca TRUPER $^{(17)}$. 


\section{Calidad seminal}

Para la evaluación de la calidad seminal, una vez transcurridos 70 días del periodo experimental ( $8 \pm 0.7$ meses de edad), se recolectaron seis muestras de semen por animal en cada tratamiento (dos por semana), utilizando una hembra estrogenizada como maniquí, empleando el método de vagina artificial provista de una camisa de caucho (Liner). Se introdujo agua caliente a $45^{\circ} \mathrm{C}$ y aire por insuflación a través de una válvula, para obtener la temperatura y la presión necesarias para estimular la eyaculación ${ }^{(18)}$. Una vez obtenidos los eyaculados se transportaron al laboratorio en condiciones isotérmicas $\left(35\right.$ a $\left.37^{\circ} \mathrm{C}\right)$ para su evaluación. En el laboratorio las muestras se colocaron en un baño maría a $37{ }^{\circ} \mathrm{C}$ para su inmediata evaluación.

A las muestras recolectadas se les determinó:

1) El volumen del eyaculado con un tubo recolector graduado.

2) Con el sistema CASA (ISAS®v1 (Proiser R+D, Valencia, España), se determinó la concentración espermática diluyendo $5 \mu 1$ de semen puro en $995 \mu 1$ de agua destilada (1:200); después se colocaron $9 \mu 1$ de la muestra diluida en una cámara de Bürker para su análisis. La motilidad espermática se evaluó depositando $5 \mu 1$ de muestra diluida a una concentración de $30 \times 10^{6}$ espermatozoides/ml sobre una cámara Makler ${ }^{\circledR}$ (Sefi Medical Instruments, Haifa, Israel), y se realizaron cuatro grabaciones de cada muestra; la dilución se realizó con el diluyente comercial (Triladyl ${ }^{\circledR}+$ agua destilada $+20 \%$ de yema de huevo). Los parámetros evaluados para esta variable fueron: porcentaje de motilidad total (MT), porcentaje de motilidad progresiva (MP), velocidad curvilínea $(\mu \mathrm{m} / \mathrm{s})(\mathrm{VCL})$, velocidad rectilínea $(\mu \mathrm{m} / \mathrm{s})$ (VSL), velocidad media ( $\mu \mathrm{m} / \mathrm{s}$ ) (VAP), índice de linealidad (LIN), índice de rectitud (STR), índice de oscilación (WOB), amplitud media del desplazamiento lateral de la cabeza (ALH), frecuencia de batido de la cabeza espermática (FBC).

3) La viabilidad espermática se evaluó mediante tinción con el fluorocromo SYBR-14/IP (Live/Dead® kit L-7011, Invitrogen ${ }^{\mathrm{TM}}$ ), se mezcló $1 \mu \mathrm{L}$ de SYBR-14 $(10 \mu \mathrm{M})$ y $1 \mu \mathrm{IP}(12$ $\mu \mathrm{M})$ en $100 \mu \mathrm{l}$ de muestra espermática diluida; se mantuvo durante 10 min a $37{ }^{\circ} \mathrm{C}$; posteriormente se colocaron $5 \mu \mathrm{l}$ de la muestra teñida sobre un portaobjeto precalentado a 37 ${ }^{\circ} \mathrm{C}$ para su evaluación en un microscopio de fluorescencia (CX-31, Olympus, Tokio, Japón), con una longitud de onda $488 \mathrm{~nm}$.

4) La actividad mitocondrial se determinó utilizando el fluorocromo J-C1 (153 $\mu \mathrm{M}$, Molecular Probes ${ }^{\circledR} \mathrm{T}-3168$, Invitrogen ${ }^{\mathrm{TM}}$ ), se mezcló $1 \mu \mathrm{l}$ del fluorocromo en $100 \mu \mathrm{de}$ muestra espermática diluida; se mantuvo durante $10 \mathrm{~min}$ a $37^{\circ} \mathrm{C}$; posteriormente se colocaron 
$5 \mu \mathrm{l}$ de la muestra teñida sobre un portaobjeto precalentado a $37^{\circ} \mathrm{C}$ para su evaluación en un microscopio de fluorescencia con una longitud de onda de $488 \mathrm{~nm}$.

5) La integridad de los acrosomas se evaluó con el fluorocromo FITC-PSA $(100 \mu \mathrm{g} / \mathrm{mL}, \mathrm{L}-$ 0770, Sigma-Aldrich ${ }^{\mathrm{TM}}$ ), añadiendo $5 \mu 1$ del fluorocromo en $100 \mu \mathrm{l}$ de muestra espermática diluida y se mantuvo durante $30 \mathrm{~min}$ a $37^{\circ} \mathrm{C}$, posteriormente se colocaron $5 \mu \mathrm{l}$ de la muestra incubada en un portaobjetos y se evaluaron en un microscopio de fluorescencia con una longitud de onda de $485 \mathrm{~nm}$.

Los datos obtenidos se analizaron mediante el procedimiento PROC GLM del paquete estadístico SAS 9.4 para Windows (SAS Inst. Inc. Cary NC, USA), se realizó un análisis de varianza (ANOVA) de medias repetidas para evaluar el efecto de la dieta sobre la calidad espermática y la comparación de medias por medio de la prueba de Tukey.

\section{Resultados y discusión}

\section{Comportamiento productivo}

Las ganancias diarias de peso de los animales en estudio, no presentaron diferencias $(P<0.05)$ entre tratamientos. Estas ganancias son mayores a las registradas por Madera-Solís et $a l^{(19)}$, quienes con diferentes niveles de inclusión de Leucaena leucocephala (Lam.) de Wit encontraron ganancias diarias de peso de 25, 41.67, 50 y $75 \mathrm{~g}$ en ovinos Pelibuey. Por su parte, Ríos et al ${ }^{(20)}$, registraron ganancias de 54,87 y $56 \mathrm{~g} /$ día, al evaluar el uso de Morus sp. y Gliricidia sepium Kunth ex Walp. como sustitutos del alimento concentrado para corderos en crecimiento. También se reportan ${ }^{(4)}$, ganancias diarias de 45.5, 96.3, 124.5 y $106.4 \mathrm{~g}$ con diferentes niveles de inclusión de heno de Hibiscus rosa-sinensis L. en ovinos en crecimiento. Las ganancias obtenidas en este estudio son menores a los señalados por otros $^{(16)}$, quienes registraron ganancias diarias de $229 \pm 10 \mathrm{~g}$ /día para animales PelibueyKatadin alojados en jaulas elevadas y alimentados con un concentrado comercial con un contenido de $16 \%$ de proteína cruda y heno de Cynodon nlemfuensis Vanderyst.

En la evaluación del rendimiento de la canal tampoco se encontraron diferencias $(P<0.05)$ entre los tratamientos: 50.79, 49.44 y $46.94 \%$ para los tratamientos T1, T2 y T3 respectivamente siendo similares a los registrados por Magaña-Monforte et al ${ }^{(16)}$, quienes obtuvieron un rendimiento de $49.1 \pm 0.58$ con animales cruzados de las razas PelibueyKatahdín. Los rendimientos de este estudio fueron inferiores a los encontrados en otro trabajo ${ }^{(21)}$, donde registran $52.8 \%$ de rendimiento de canal para machos enteros, $56.64 \%$ para machos castrados y 55.87 para hembras. El adecuado balance nutricional presente en las dietas evaluadas generó una respuesta productiva similar en los animales de estudio y adecuada al compararse con otros trabajos realizados en condiciones similares. Estos 
resultados en las raciones evaluadas (Cuadro 2) pueden ser atribuidos al adecuado nivel de proteína y al mejor balance nutricional ${ }^{(22)}$, lo que estimula un aumento en el nivel de eficiencia de los nutrimentos producida por una mayor actividad microbial en el rumen ${ }^{(23)} \mathrm{y}$ garantizando con esto una estabilidad metabólica ${ }^{(24)}{ }^{2}$ afectando positivamente los diferentes parámetros productivos evaluados en el presente estudio (ganancias diarias de peso y rendimiento de la canal).

\section{Desarrollo testicular}

El volumen testicular es un indicador de la capacidad fecundante del macho, la fertilidad más alta, así como un menor número de espermatozoides anormales se ha observado en animales con mayor circunferencia escrotal. En los animales de estudio el desarrollo testicular determinado por medio del incremento de la circunferencia y el diámetro escrotal, presentaron diferencias $(P<0.05)$ entre los tratamientos evaluados para ambas variables (Cuadro 3). Para la variable circunferencia escrotal el tratamiento T3 mostró la mayor media con respecto al T2, mientras que el T1 no difirió $(P>0.05)$ con los otros dos tratamientos. Para la variable de diámetro escrotal, no existió diferencia entre los tratamientos T1 y T3, pero ambos fueron mayores con respecto a $\mathrm{T} 2$.

Cuadro 3: Circunferencia escrotal, diámetro escrotal, volumen y concentración seminal de ovinos de pelo suplementados con $M$. oleifera y $T$. gigantea (media \pm error estándar)

\begin{tabular}{clllll}
\hline Tratamientos & $\begin{array}{l}\text { Circunferencia } \\
\text { escrotal }(\mathbf{c m})\end{array}$ & $\begin{array}{l}\text { Diámetro } \\
\text { escrotal } \\
(\mathbf{m m})\end{array}$ & $\begin{array}{l}\text { Volumen } \\
(\mathbf{m l})\end{array}$ & Concentración \\
\hline $\mathrm{T} 1$ & $26.48 \pm 0.33^{\mathrm{ab}}$ & $48.84 \pm 1.05^{\mathrm{a}}$ & $\begin{array}{l}0.70 \\
0.05^{\mathrm{a}}\end{array}$ & \pm & $1882.63 \pm 202.66^{\mathrm{a}} \times 10^{6}$ \\
& & & 0.61 & \pm & $1841.92 \pm 182.34^{\mathrm{a}} \times 10^{6}$ \\
$\mathrm{~T} 2$ & $25.42 \pm 0.33^{\mathrm{b}}$ & $44.57 \pm 1.05^{\mathrm{b}}$ & $0.05^{\mathrm{a}}$ & & \\
& & & 0.63 & \pm & $1014.49 \pm 482.44^{\mathrm{a}} \times 10^{6}$ \\
$\mathrm{~T} 3$ & $26.62 \pm 0.33^{\mathrm{a}}$ & $48.83 \pm 1.05^{\mathrm{a}}$ & $0.06^{\mathrm{a}}$ & & \\
\hline
\end{tabular}

T1: dieta integral con $40 \%$ de M. oleifera + pasto Taiwán (Pennisetum purpureum), T2: dieta integral con 40\% de T. gigantea + pasto Taiwán, y T3: alimento comercial + pasto Taiwán.

$\left({ }^{\mathrm{a}, \mathrm{b}}\right)$ Literales distintas entre columnas indican diferencias significativas $(P<0.05)$.

Las medias para la circunferencia escrotal fueron: $26.4 \pm 0.33,25.4 \pm 0.33$ y $26.6 \pm 0.33 \mathrm{~cm}$ para los tres tratamientos uno dos y tres, respectivamente (Cuadro 3), son menores a los registrados por Luna et $a l^{(25)}$, quienes encontraron medias de $28.4 \mathrm{~cm}$ en ovinos suplementados con grasa animal y aceite de palma; al igual que los reportados en otros trabajos ${ }^{(26,27)}$, con 29.4 y $36.5 \mathrm{~cm}$, respectivamente. Las medias de diámetro escrotal fueron menores a las registradas por Ballín et al ${ }^{(26)}$. El desarrollo testicular está determinado por 
diferentes factores, principalmente la edad, la raza y la nutrición ${ }^{(27)}$, en este sentido los animales en estudio con una edad promedio de $7 \pm 0.5$ meses de edad, recibieron una dieta con niveles adecuados de proteína y además nutrientes necesarios para cubrir los requerimientos nutricionales de acuerdo a su etapa fisiológica, lo cual se refleja en las ganancias de peso y el desarrollo testicular obtenido.

\section{Evaluación seminal}

Las variables de volumen del eyaculado y la concentración espermática no presentaron diferencias $(P>0.05)$, entre tratamientos (Cuadro 3). El volumen en los tres tratamientos se encuentra dentro de los rangos considerados como normales, que van de 0.5 a $2.0 \mathrm{ml}$, y este volumen varía según la edad, tamaño, condición corporal del animal, frecuencia de recolección y destreza del operador ${ }^{(28)}$. A pesar de esto, los valores reportados en este experimento fueron menores a los reportados por Mansano et al ${ }^{(29)}$, quienes obtuvieron volúmenes de $1.3 \pm 0.4 \mathrm{ml}$; Carrillo y Hernández ${ }^{(30)}$ obtuvieron $1.41 \pm 0.11 \mathrm{ml}$, por su parte Córdova-Izquierdo et al $^{(31)}$ obtuvieron $1.11 \mathrm{ml}$; sin embargo, estos dos trabajos se realizaron con machos maduros a diferencia de los machos púberes ( $7 \pm 0.5$ meses de edad) utilizados en el presente estudio.

Los valores bajos de volumen del eyaculado obtenidos en este estudio, contienen una mayor concentración de espermatozoides por $\mathrm{ml}$, por lo tanto, estos valores son superiores a los registrados en otros estudios, quienes indican rangos de 206.4 a $711.89 \times 10^{6(30,31)}$, pero son similares a los encontrados por Domínguez et $a l^{(32)}$, quienes registraron valores de $2.19 \pm$ 0.04 y $108 \pm 8.0 \times 10^{6}$ espermatozoides $/ \mathrm{ml}$, al evaluar la calidad seminal de corderos (F1) Katahdin $\times$ Pelibuey suplementados con alfalfa. En contraste los valores de concentración obtenidos aquí son menores a los encontrados por Quintero et al ${ }^{(33)}$, quienes obtuvieron $2,628.2 \times 10^{6}$ al evaluar ovinos de pelo, de 2 a 5 años de edad.

En los parámetros de motilidad se encontraron diferencias $(P<0.05)$, en motilidad total, velocidad curvilínea y ALH, donde los tratamientos T1 y T2 fueron mejores vs T3; y en las variables de VAP y VSL, el T1 presentó los mejores resultados respecto al T3, pero no se observaron diferencias entre T1 y T2. Para el resto de las variables analizadas (MP, LIN, STR, WOB y BCF) no se encontraron diferencias entre grupos experimentales (Cuadro 4). 
Cuadro 4: Parámetros de motilidad seminal de ovinos de pelo suplementados con $M$. oleifera y $T$. gigantea (media \pm error estándar)

\begin{tabular}{|c|c|c|c|}
\hline \multirow{2}{*}{ Variables } & \multicolumn{3}{|c|}{ Tratamientos } \\
\hline & T1 & T2 & T3 \\
\hline MT, \% & $93.9 \pm 2.1^{\mathrm{a}}$ & $88.6 \pm 1.9^{\mathrm{a}}$ & $71.9 \pm 4.0^{b}$ \\
\hline $\mathrm{VAP}, \mu \mathrm{m} / \mathrm{s}$ & $100.4 \pm 3.7^{\mathrm{a}}$ & $88.6 \pm 3.2^{\mathrm{ab}}$ & $73.0 \pm 6.8^{b}$ \\
\hline $\mathrm{VCL}, \mu \mathrm{m} / \mathrm{s}$ & $157.8 \pm 5.0^{\mathrm{a}}$ & $146.6 \pm 4.4^{\mathrm{a}}$ & $117.3 \pm 9.2^{b}$ \\
\hline $\mathrm{VSL}, \mu \mathrm{m} / \mathrm{s}$ & $70.3 \pm 3.1^{\mathrm{a}}$ & $61.3 \pm 2.7^{\mathrm{ab}}$ & $54.6 \pm 5.8^{\mathrm{b}}$ \\
\hline $\mathrm{ALH}, \mu \mathrm{m}$ & $4.8 \pm 0.1^{\mathrm{a}}$ & $4.6 \pm 0.1^{\mathrm{a}}$ & $3.7 \pm 0.3^{b}$ \\
\hline $\mathrm{MP}, \%$ & $30.79 \pm 2.64^{\mathrm{a}}$ & $29.23 \pm 2.32^{\mathrm{a}}$ & $30.18 \pm 4.87^{\mathrm{a}}$ \\
\hline STR, \% & $64.72 \pm 1.31^{\mathrm{a}}$ & $64.98 \pm 1.15^{\mathrm{a}}$ & $69.62 \pm 2.42^{\mathrm{a}}$ \\
\hline WOB, $\%$ & $61.96 \pm 1.63^{\mathrm{a}}$ & $59.85 \pm 1.43^{\mathrm{a}}$ & $60.78 \pm 3.01^{\mathrm{a}}$ \\
\hline $\mathrm{BCF}, \mathrm{Hz}$ & $10.32 \pm 0.25^{\mathrm{a}}$ & $10.57 \pm 0.22^{\mathrm{a}}$ & $9.89 \pm 0.47^{\mathrm{a}}$ \\
\hline LIN, \% & $42.27 \pm 1.9^{\mathrm{a}}$ & $40.85 \pm 1.67^{\mathrm{a}}$ & $44.35 \pm 3.5^{\mathrm{a}}$ \\
\hline
\end{tabular}

T1 $=40 \%$ de $M$. oleifera + Taiwán, T2=40\% de $T$. gigantea + Taiwán, T3= alimento comercial + Taiwán. $\mathrm{MT}=$ motilidad total; $\mathrm{VAP}=$ velocidad media; $\mathrm{VCL}=$ velocidad curvilínea; $\mathrm{VSL}=$ velocidad rectilínea; $\mathrm{ALH}=$ amplitud de desplazamiento; $\mathrm{MP}=$ motilidad progresiva; $\mathrm{STR}=$ índice de rectitud; $\mathrm{WOB}=$ índice de oscilación; $\mathrm{BCF}=$ frecuencia de batido; $\mathrm{LIN}=$ índice de linenealidad.

${ }^{\mathrm{a}, \mathrm{b}}$ Valores con distinta literal en la misma fila son diferentes $(P<0.05)$.

Los resultados en la variable de motilidad son superiores a los registrados por Mansano et $a l^{(29)}$, quienes obtuvieron $52 \%$ motilidad, pero son similares a los encontrados en otra investigación ${ }^{(32)}$, donde encontraron $80 \%$ de motilidad total y motilidad progresiva de más de $20 \%$; sin embargo, los resultados de este estudio son menores a los registrados por Quintero et $a l^{(33)}$, quienes obtuvieron $90.69 \%$ de motilidad progresiva.

En el análisis de la variable de viabilidad de la membrana plasmática se encontraron diferencias $(P<0.05)$ entre los tratamientos evaluados (T1: $54 \%$, T2: $63 \%$ y T3: $21 \%)$ siendo el tratamiento T2 el que presentó mayor porcentaje de viabilidad, en contraste con T3 que obtuvo los porcentajes más bajos (Figura 1). 
Figura 1: Viabilidad, actividad mitocondrial e integridad del acrosoma de ovinos de pelo suplementados con Moringa oleífera y Trichanthera gigantea

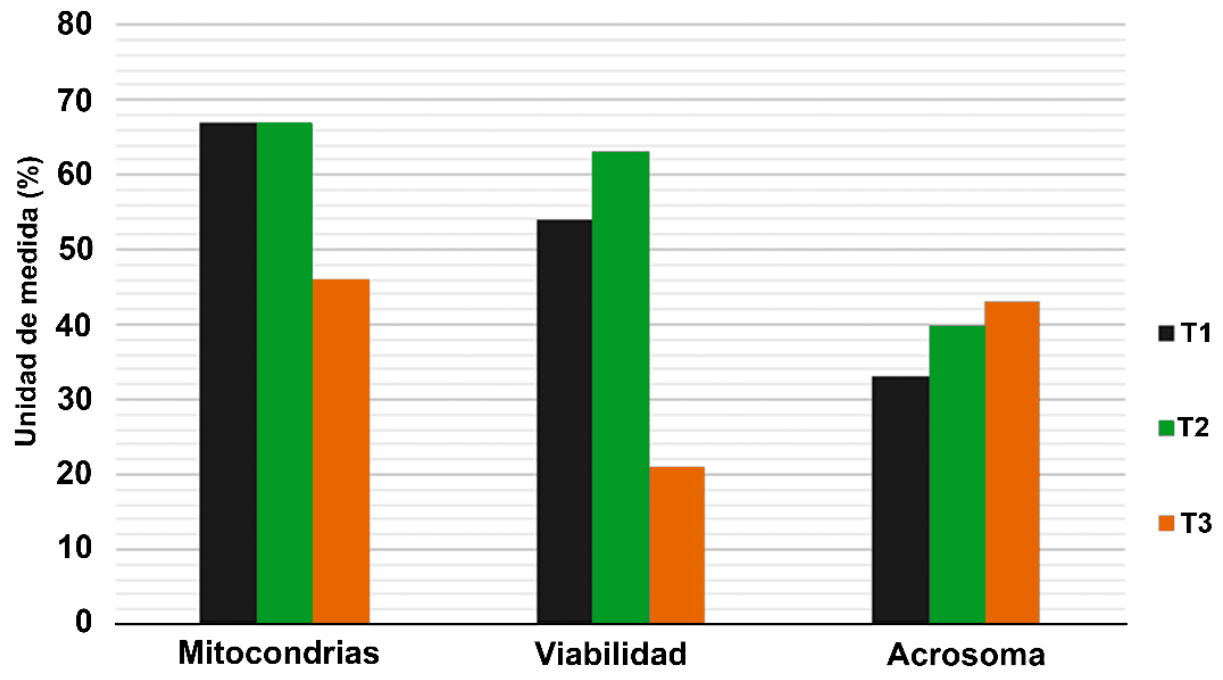

T1: dieta integral con 30\% de M. oleifera + pasto Taiwán (Pennisetum purpureum Schumach.), T2: dieta integral con 30\% de T. gigantea + pasto Taiwán, y T3: alimento comercial + pasto Taiwán.

Las variables actividad mitocondrial e integridad del acrosoma no presentaron diferencias $(P>0.05)$ entre ninguno de los tratamientos; aunque para la variable de actividad mitocondrial en el T1 presentó las medias más elevadas, mientras que el T3 presentó los mayores porcentajes para la variable actividad mitocondrial (Figura 1). La integridad de las membranas plasmática y acrosomal así como la actividad mitocondrial son un factor primordial para la fecundación, ya que una membrana plasmática intacta indica que el espermatozoide está vivo, una membrana acrosomal intacta permite la penetración del ovocito y la actividad mitocondrial asegura la presencia de energía necesaria para el desplazamiento del espermatozoide (Figura 2), por ello su inclusión en las evaluaciones sobre calidad seminal es imprescindible ${ }^{(31)}$. Los resultados observados en este estudio concuerdan con los registrados por Córdova-Izquierdo et $\mathrm{l}^{(31)}$ quienes obtuvieron $68.5 \%$ de integridad de la membrana plasmática al evaluar ovinos de la raza Suffolk. Asimismo, coinciden con los resultados de Rubio-Guillen $e t a l^{(34)}$, quienes registran $42 \%$ al evaluar ovinos West African. Sin embargo, los porcentajes obtenidos en este estudio son menores a los encontrados en otras investigaciones ${ }^{(30,32,33)}$; pero se concuerda con Domínguez et al ${ }^{(32)}$ en los valores de la integridad acrosomal. 
Figura 2: Espermatozoides de ovinos alimentados con Trichanthera gigantea o Moringa

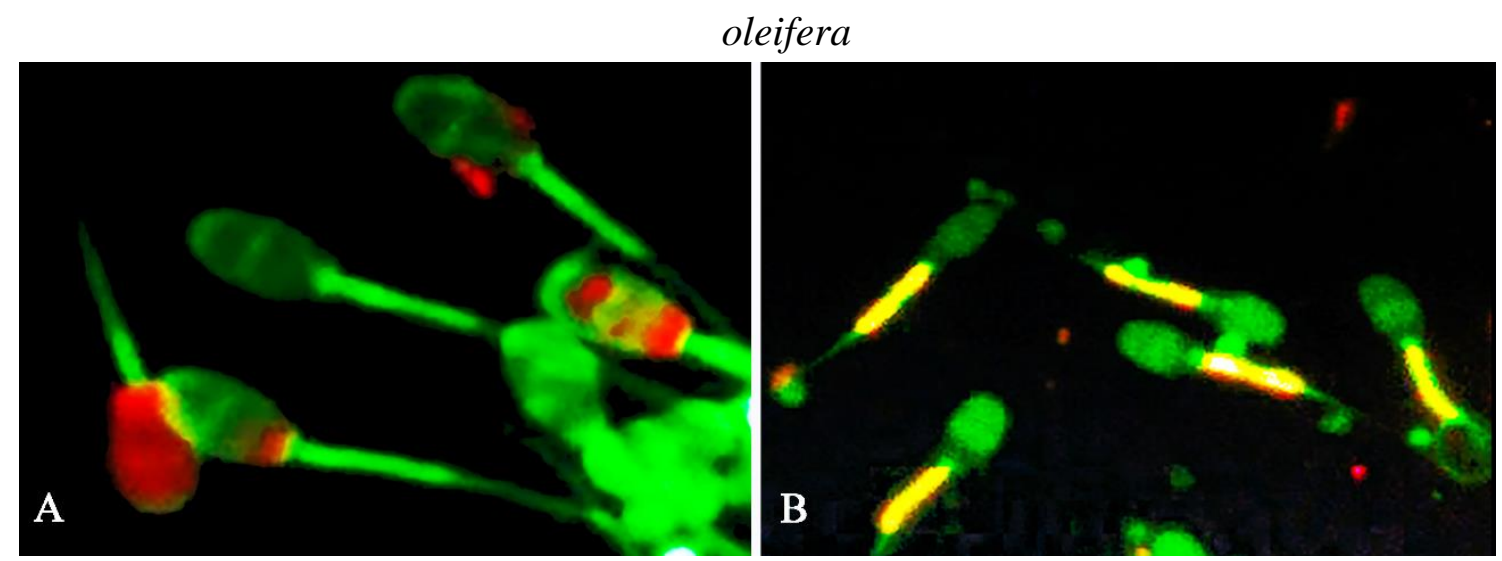

A. Espermatozoides teñidos con fluorocromos SYBER-14/IP, vivos (cabezas verdes) y muertos (cabezas rojas o naranjas); viabilidad del acrosoma ubicado en la región apical mediante el fluorocromo FITC-PSA (incolora viable y verde dañada). B. Actividad mitocondrial ubicado en la región intermedia de la cola teñidos con el fluorocromo JC1, con actividad (naranjas o amarillos) sin actividad (verdes).

Los parámetros registrados en la evaluación de la calidad seminal, así como el desarrollo testicular podrían atribuirse al adecuado balance nutricional de las dietas evaluadas, ya que la producción y la calidad espermática, así como el tamaño testicular están directamente influenciados por la nutrición. Un aumento en el nivel nutricional incrementa la frecuencia de pulsos de LH e incrementa los niveles de $\mathrm{FSH}^{(35)}$, hormonas que promueven la espermatogénesis y la secreción de andrógenos ${ }^{(36)}$.

Los animales evaluados en el presente estudio son animales púberes, y animales en esta condición presentan una mayor cantidad de espermatozoides inmaduros, muertos o defectuosos, que a su vez producen altas cantidades de radicales libres que afectan a los espermatozoides que se encuentran en buen estado, ya que los radicales libres provocan una cascada de peroxidación lipídica afectando principalmente ácidos grasos no saturados de la membrana plasmática del espermatozoide, causando pérdida de la función espermática y apoptosis celular, lo cual los coloca entre las principales causas de muerte en las células espermáticas $^{(37)}$.

Se ha observado que la inclusión de antioxidantes en la dieta mejora el porcentaje de células espermáticas vivas, debido a que los antioxidantes eliminan radicales libres que se encuentran en el plasma seminal y confieren protección a los espermatozoides ${ }^{(13,14)}$; Priyadarshani y Varma ${ }^{(38)}$, observaron un mayor porcentaje de células vivas cuando utilizaban harina de $M$. oleifera en la dieta de ratones. Esto es similar a lo observado en el presente estudio y podría atribuirse al aporte de antioxidantes ( $\beta$-carotenos) que contienen M. oleifera y T. gigantea ${ }^{(15)}$. 
Los compuestos secundarios presentes en las dos especies evaluadas podrían estar relacionados con los valores encontrados en el presente estudio, por lo tanto, estudios que determinen la presencia y la concentración de estos aportaría mayor evidencia al respecto.

\section{Conclusiones e implicaciones}

La sustitución de $40 \%$ de alimento comercial por M. oleifera o $T$. gigantea en la dieta de ovinos Pelibuey promueve un desarrollo testicular adecuado, mejora la viabilidad espermática en más de $20 \%$ y algunos parámetros de motilidad como la motilidad total, velocidad media, velocidad curvilínea, velocidad rectilínea y la amplitud media del desplazamiento lateral de la cabeza espermática, lo que podría mejorar el potencial reproductivo de los sementales.

\section{Agradecimientos}

Este trabajo fue financiado parcialmente por el proyecto CONACYT Ciencia Básica 164592 y por el proyecto 5304.14-P del Tecnológico Nacional de México. Agradecemos las sugerencias y comentarios al manuscrito por parte de los revisores anónimos.

\section{Literatura citada:}

1. Altieri MC, Nicholls CI. Agroecología. Teoría y práctica para una agricultura sustentable. México: Programa de las Naciones Unidas para el Medio Ambiente Red de Formación Ambiental para América Latina y el Caribe; 2000.

2. Ramírez MEI. Productive response of pelibueysheep diet supplemented with Moringa oleifera Lam. (Moringaceae) and Trichanthera gigantea (Bonpl.) Nees (Acanthaceae). Int J Agric Sci 2017;8(2):172-176.

3. Galindo JD. Impacto de los árboles, los arbustos y otras leguminosas en la ecología ruminal de animales que consumen dietas fibrosas. Pastos y Forrajes 2005;28:59-67.

4. Ruiz-Sesma DL. Evaluación nutritiva y productiva de ovinos alimentados con heno de Hibiscus rosa-sinensis. Zootec Trop 2006;24:467-482.

5. Apráez JE, Delgado JM, Narváez J. Composición nutricional, degradación in vitro y potencial de producción de gas, de herbáceas, arbóreas y arbustivas encontradas en el trópico alto de Nariño. Livest Res Rural Dev 2012;24(3): http://www.lrrd.org/lrrd24/3/apra24044.htm. Consultado 10 Feb, 2018. 
6. Katsube TT, Tsurunaga Y, Sugiyama M, Furuno T, Yamasaki Y. Effect of air-dryng temperature on antioxidant capacity and stability of polyphenolic compunds in mulberry (Morus alba L.) leaves. Food Chem 2009;113(4):964-969.

7. Sosa RE. Evaluación del potencial forrajero de árboles y arbustos tropicales para la alimentación de ovios. Téc Pecu Méx 2004;42(2):129-144.

8. Carmona J. Efecto de la utilización de árboreas y arbustivas forrajeras sobre la dinámica digestiva en bovinos. Rev Lasallista Investig 2007;4(1):40-50.

9. Rivera LJ, Ramon UJ. Factores antinutricionales del recursos forrajero disponible en Yucatán como una posibilidad para mitigar el efecto de los gases invernadero causados por los rumiantes. In: Dumonteil E editor. Contribución de la biotecnología al desarrollo de la Peninsula de Yucatán. Mérida: Fondo Mixto CONACYT-Gobierno del Estado de Yucatán. 2012;99-115.

10. Lenis YG. Efectos de los fitoestrógenos en la reproducción animal. Rev Fac Nac Agron 2010;63(2):5555-5565.

11. Retana-Márquez S, Hernández H, Flores JA, Muñoz-Gutiérrez M, Duarte G, Vierlma J, Fitz-Rodríguez G, Fernández IG, Keller M, Delgadillo JA. Effects of phytoestrogens on mammalian reproductive physiology. Trop Subtrop Agroecosyt 2012;15:129-145.

12. García D. Evaluación química de especies no leguminosas con potencial forrajero en el estado de Trujillo Venezuela. Zootec Trop 2006;24:401-415.

13. Balercia G, Armeni T, Mantero F, Principato G, Regoli F. Total oxyradical scavenging capacity toward different reactive oxygen species in seminal plasma and sperm cells. Clin Chem Lab Med 2003;41(1):13-19.

14. Raijmakers MT, Roelofs HM, Steegers EA, Steegers-Theunissen RR, Mulder TP, Knapen MF, Wong WY, Peters WH. Glutathione and glutathione S-transferases A1-1 and $\mathrm{P} 1-1$ in seminal plasma may play a role in protecting against oxidative damage to spermatozoa. Fertil Steril 2003;79(1):169-172.

15. Ghasi S, Nwobobo E, Ofili JO. Hypocholesterolemic effects of crude extract of leaf of Moringa oleifera Lam. in high-fat diet fed wistar rats. J Ethnopharmacol 2000;69(1):2125.

16. Magaña-Monforte JG, Moo-Catzin CJ, Chay-Canul JR, Aké-López JR, Segura-Correa JC, Montés-Pérez RC. Crecimiento y componentes de la canal de ovinos de pelo en jaulas elevadas. Livest Res Rural Dev 2015;27(6).

17. Jiménez-Severiano HRP. Evaluation of mathematical models to desribe testicular growth in Blackbelly ram lambs. Theriogenology 2010;74(7):117-1114. 
18. Ruiz LS. Evaluación de la calidad espermática del semen ovino posdescongelación al emplear dos fuentes energéticas y dos crioprotectores. Rev Inv Perú 2015;26(1):49-56.

19. Madera-Solís NB, Bacab-Pérez HM, Ortiz De La Rosa B. Ganancia diaria de peso en ovinos por inclusión de una planta leguminosa (Leucaena leucocephala) en dietas basadas en pasto clon Cuba CT115 (Pennisetum purpureum). Bioagrociencias 2013;6(1):24-31.

20. Ríos L, Rondón Z, Combellas J, Alvarez R. Uso de morera (Morus sp.) y mata ratón (Gliricidia sepium) como sustituto del alimento concentrado para corderos en crecimiento. Zootec Trop 2005;23:49-60.

21. Torrescano GR, Sánchez A, Peñúñuri FJ, Velázquez J, Sierra T. Características de la canal y calidad de la carne de ovinos pelibuey, engordados en Hermosillo, Sonora. BIOtecnia 2009;11(1):41-50.

22. González-González NN, Gutiérrez-González D, García-López R, Fernández-Mayer A. Consumo voluntario y valores metabólicos sanguíneos en caprinos alimentados con mezclas integrales frescas de Moringa oleifera: Penisetum purpureum Clon-OM22. Avances en Investigación Agropecuaria 2015;19:25-36.

23. Garza-F JD, Owens FN, Welty S. Effect of post-ruminal protein infusion on feed intake and utilization of low quality hay by beef steers. Anim Sci Pap Rep 1991;134:106-113.

24. Borroto-Pérez A, Negrín-Brito A, Peña-López P, Vega-Báez D. Uso de moringa (Moringa oleifera) para ovinos en crecimiento, como alternativa alimentaria ambientalmente amigable. Universidad \& Ciencia 2018;7:78-90.

25. Luna C, Aguilar JA, Peralta JA, Velázquez JR. Efecto del aceite de palma sobre el crecimiento y capacidad reproductiva de carneros de pelo púberes. Arch Zootec 2013;62(237):45-52.

26. Ballín FJ, Ochoa MA, Torres G, Morón FJ, Gonzáles JM, Díaz MO. Relación de la edad, peso corporal y medidas morfométricas sobre el inicio de la pubertad en corderos polypay del altiplano potosino. Revista Cientifica LUZ 2013;23:434-439.

27. Moreno-Cañez E, Ortega-García C, Cáñez-Carrasco MG, Peñúñuri-Molina F. Evaluación del comportamiento posdestete en corral de futuros sementales ovinos de raza Katahdin y Pelibuey en Sonora. TECNOCIENCIA Chihuhua 2013;7(1):7-16.

28. Aisen EG. Reproducción ovina y caprina. Buenos Aires: Intermedica; 2004.

29. Mansano MM, Scott C, Souza MD, Torre LT, Vallejo AV, Ferreira SF. Viabilidad de espermatozoides ovinos mantenidos a $5^{\circ}$ y $15^{\circ} \mathrm{C}$ en diferentes sistemas de refrigeración. Revista Brasileira de Ciência Veterinária 2014;21(2):122-126. 
30. Carrillo GD, Hernández HD. Caracterización seminal de individuos ovinos criollos colombianos de pelo en el departamento de Sucre. Rev Colombiana Cienc Anim 2016;8(2):197-203.

31. Córdova-Izquierdo A, Saltijeral-Oaxaca J, Muñoz-Mendoza R, Córdova-Jiménez MS, Cordova -Jimenez CA, Guerra-Liera JE. Efecto del método de obtención de semen ovino sobre la calidad espermática. Rev Electron Vet 2006;7(8):1-5.

32. Domínguez-Rebolledo AE, Alcaraz-Romero A, Cantón-Castillo JG, Loeza-Concha H, Ramón-Ugalde J. Efecto de la alfalfa (Medicago sativa L.) en la dieta sobre la calidad de los espermatozoides epididimarios de ovinos Katahdin con pelibuey. Reunión científica tecnológica forestal y agropecuaria Tabasco 2014 y III Simposio internacional en producción agroalimentaria tropical. Tabasco: Instituto Nacional de Investigaciones Forestales, Agrícolas y Pecuarias 2014:186-189.

33. Quintero EJA, Clemente SF, Olguín AHA. Contribución en el desarrollo de un índice de calidad del semen para la valoración de sementales ovinos. CULCYT 2016;58(13):105109.

34. Rubio-Guillen J, Quintero-Montero AA, González-Villalobos DM. Efecto de la criopreservación sobre la integridad de la membrana plasmática y acrosomal de espermatozoides de toros. Rev Cient (Maracaibo) 2009;19(4):382-389.

35. Blanche DZ. Fertility in males: modulators of the acute effects of nutrition on the reproductive axis. Reprod Suppl 2003;59:219-233.

36. Prieto-Gómez B, Velázquez-Paniagua M. Fisiología de la reproducción: hormona liberadora de gonadotrofinas. Rev Fac Med UNAM 2002;45(6):252-257.

37. Lozano H. Factores que afectan la calidad seminal en toros. Rev Med Vet Zoot 2009;56:258-272.

38. Priyadarshani N, Varma MC. Effect of Moringa oleifera leaf poder on sperm count, histology of testis and epididymis of hyperglycaemic mice Mus musculus. Am Int J Res Form Appl Nat Sci 2007;14:7-13. 